\title{
Stereotactic radiosurgery for spinal metastases delivered with volumetric modulated arc therapy - a new era in radiosurgery
}

\author{
Song $\mathrm{Y}^{1 *}$, Mueller $\mathrm{B}^{2}$, Voros $\mathrm{L}^{1}$, Zhang $\mathrm{M}^{1}$, Tang $\mathrm{X}^{1}$ and Mychalczak $\mathrm{B}^{2}$ \\ ${ }^{1}$ Department of Medical Physics, Memorial Sloan Kettering Cancer Center, New York, USA \\ ${ }^{2}$ Department of Radiation Oncology, Memorial Sloan Kettering Cancer Center, New York, USA
}

\begin{abstract}
Primary spinal tumors are rare. The vast majority of spinal tumors are metastases. With the advent of volumetric modulated arc therapy (VMAT) and rapid improvement in image-guided technology, VMAT stereotactic radiosurgery (SRS) is becoming the preferred treatment option for patients with spinal metastases. In this paper, we present this novel clinical technology as implemented in our institution..
\end{abstract}

\section{Introduction}

More than $90 \%$ of spinal tumors are distant metastases. Nearly $50 \%$ of all cancer patients will develop spinal metastases, primarily via the route of hematogenous dissemination [1]. This is the deadliest phase of cancer progression. There are nearly 200,000 new cases each year in the U.S. Many spinal metastases present with associated epidural disease that presents a special problem. As such lesions in the spine grow, they can compress the spinal cord, the cauda equina, and the nerve roots, resulting in intractable pain, loss of motor/nerve function, and even paralysis. Bone fracture and mechanical instability of the spine are additional sequelae from spinal metastases that can lead to disabling pain and function [2]. The common treatment options for spinal metastases are radiotherapy, surgery, systemic therapy, and lately, stereotactic radiosurgery [3]. In this paper, we present a novel approach to the treatment of spinal metastases with stereotactic radiosurgery (SRS) delivered with volumetric modulated arc therapy (VMAT).

\section{Material and method}

A young female patient with triple negative breast cancer complained of low back pain. Lumbar spine MRI showed diffuse abnormal enhancement in the L2 vertebral body with mild cord compression and deformity of the vertebral end plates and mild paraspinal extension anteriorly. A CT-guided biopsy confirmed a metastasis in the L2 vertebral body. She was subsequently treated with VMAT spinal SRS using a single fraction of 2400 cGy. For SRS treatment planning, the patient was CT-simulated on an SRS board in a supine position with hands folded across the chest. The planning target volume (PTV) consisted of the L2 vertebral body including bilateral transverse processes, the inferior end plate of the L1 vertebral body, and the superior end plate of the L3 vertebral body, which formed a circumferential PTV around the vertebral foramen (Figures 1-3). The organs at risk (OAR) included the bowel, cauda equina, thecal sac, spinal cord, liver, and bilateral kidneys. In addition, a series of optimization structures were also contoured for dose tuning. By assigning proper dose constraints, these tuning structures could drive the higher isodose

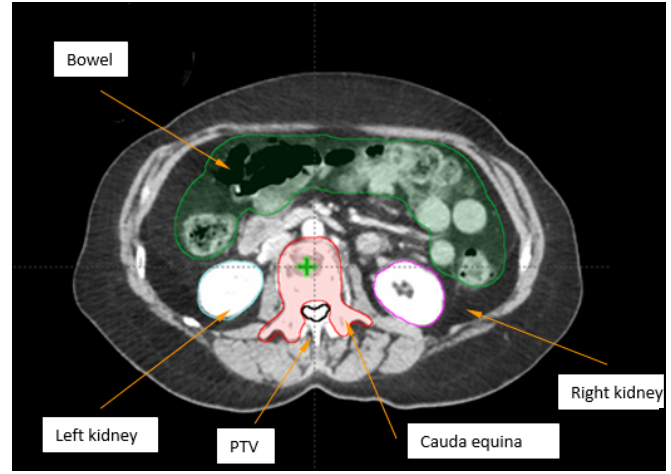

Figure 1. Planning target volume (PTV) and organs at risk (OAR) shown in a CT axial plane

curves closer to the PTV, thus eliminating the undesirable hot spots in normal tissue and reducing integral dose to the body as well.

\section{Result}

The VMAT spinal SRS plan composed of four posterior short arcs with a span of $84^{\circ}$. The treatment isocenter was placed at the geometric center of L2 vertebral body to minimize treatment field sizes and multileaf collimator (MLC) dose leakage (Figure 4). The arc control points were interleaved in gantry angles, which augmented the dynamic range of intensity modulation. The collimator angles were alternated between $0^{\circ}$ and $90^{\circ}$ to further improve intensity modulation. Although $360^{\circ}$ full arcs with a $190^{\circ}$ anterior avoidance sector could be used, this approach

*Correspondence to: Song Y, Faculty of Medical Physics, Department of Medical Physics, Memorial Sloan Kettering Cancer Center Bergen, Summit Ave, Montvale, USA, E-mail: songy@mskcc.org

Key words: Radiosurgery, SRS, VMAT, Spinal Metastasis

Received: April 09, 2018; Accepted: May 10, 2018; Published: May 14, 2018 


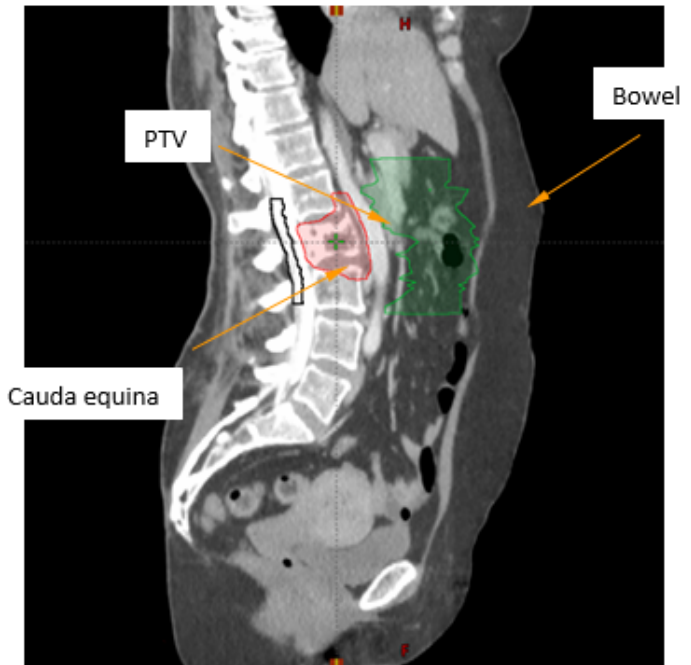

Figure 2. Planning target volume (PTV) and organs at risk (OAR) shown in a CT sagittal plane

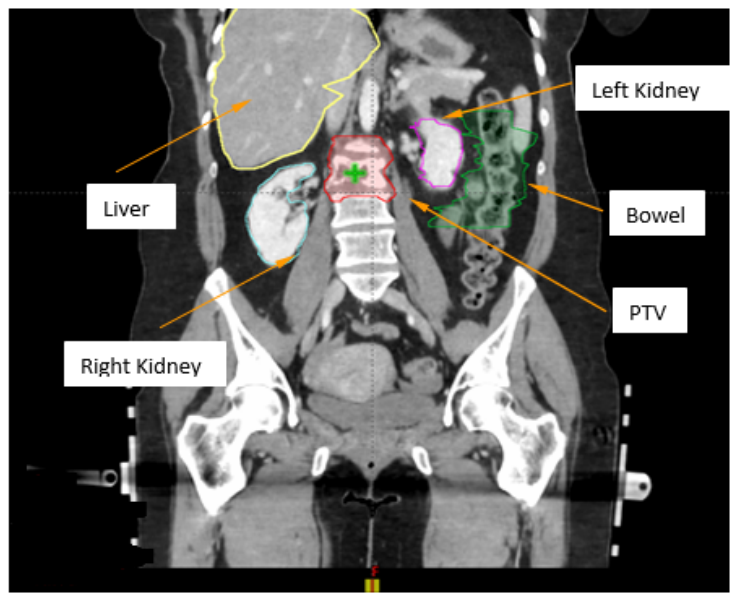

Figure 3. Planning target volume (PTV) and organs at risk (OAR) shown in a CT corona plane

would significantly reduce the control point density, resulting in an inferior plan. The jaws were fitted to beam apertures to mitigate MLC dose leakage. The plan was optimized with a low dose constraint and a high priority on the spinal cord.

Figure 5 shows the dose distribution in a representative coronal plane. The green line represents the $100 \%$ isodose line. All the dosimetric parameters met our institutional dose tolerances. In particular, the PTV dose conformity index was 1.04 . The PTV V ${ }_{100 \%}$ was $96 \%$, with a mean PTV dose of $2563.4 \mathrm{cGy}$. The maximal and mean cord doses were 816.5 and $191.9 \mathrm{cGy}$, respectively, far below our institutional dose tolerance of $1400 \mathrm{cGy}$. The VMAT spinal SRS plan was delivered on a Varian TrueBeam machine using a $6 \mathrm{X}$ FFF beam with an intra-fractional KV imaging frequency of one image $/ 20^{\circ}$.

\section{Discussion and conclusion}

Recent studies have demonstrated that spinal SRS is an effective treatment for patients with oligometastatic disease and without spinal instability [4]. Spinal SRS is also a preferred treatment for radioresistant tumor histologies such as renal cell carcinoma, melanoma, sarcoma, hepatocellular and colorectal cancers. Such tumors require large radiation fraction sizes to achieve durable local control. In addition, spinal SRS is the preferred treatment option in the setting of tumor recurrence after failed conventional radiotherapy. This is an effective strategy to limit radiation dose to the spinal cord. Recently, the combination of percutaneous vertibroplasty or kyphoplasty and postoperative adjuvant SRS has also become a new treatment paradigm for spinal metastases. Clinical data shows that spinal SRS is correlated with higher rates of fusion and lower rates of hardware failure.

Traditionally, spinal SRS had been delivered using either dynamic conformal arcs (DCA) or intensity modulated radiation therapy (IMRT). However, DCA does not have the necessary intensity modulation to produce a steep dose gradient between the target and spinal cord interface. Although IMRT has eliminated this technical deficiency, the inherent limitation of static fields makes it difficult to produce a conformal avoidance required for the treatment of circumferential targets around the spinal cord. VMAT, as a novel rotational modality, has transcended this dosimetric barrier and become the preferred technique for spinal SRS.

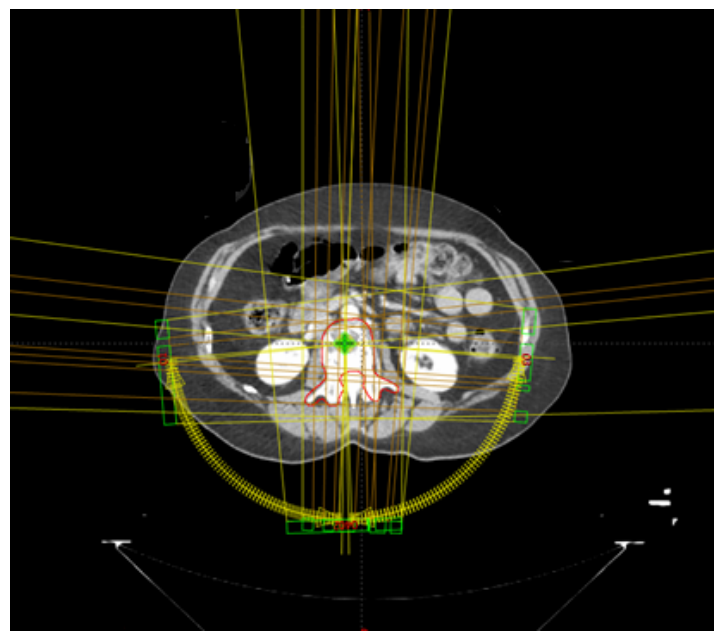

Figure 4. The VMAT spinal SRS plan consisted of 4 posterior arcs, as indicated by yellow arc sectors. The yellow arrows indicate the arc rotation directions. The green cross inside the $\mathrm{L} 2$ vertebral body represents the plan treatment isocenter.

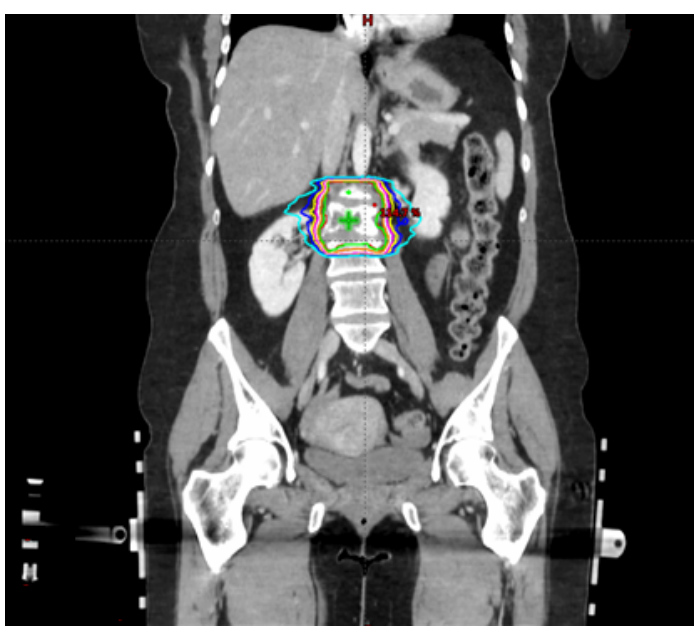

Figure 5. The isodose distribution of the VMAT spinal SRS plan. The green line represent the $100 \%$ isodose line. It is highly conformal to the shape of PTV. 


\section{References}

1. Cancer Statistics Facts: Lung and Bronchus Cancer, Surveillance, Epidemiology, and End Results Program (SEER), National Cancer Institute.

2. Coleman RE (2006) Clinical features of metastatic bone disease and risk of skeletal morbidity. Clin Cancer Res 12: 6243s-62492. [Crossref]
3. NCCN (2018) Clinical Practice Guidelines in Oncology, National Comprehensive Cancer Network (NCCN).

4. Palma DA, Haasbeek CJ, Rodrigues GB, Dahele M, Lock M, et al. (2012) Stereotactic ablative radiotherapy for comprehensive treatment of oligometastatic tumors (SABRCOMET): study protocol for a randomized phase II trial. BMC Cancer 12: 305-312. [Crossref]

Copyright: (C2018 Song Y. This is an open-access article distributed under the terms of the Creative Commons Attribution License, which permits unrestricted use, distribution, and reproduction in any medium, provided the original author and source are credited. 